\title{
SIGNATURE OF SQUEEZING IN CONTROLLED QUANTUM SYSTEMS
}

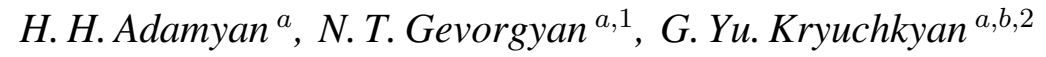 \\ ${ }^{a}$ Institute for Physical Research, National Academy of Sciences, Ashtarak-2, Armenia \\ ${ }^{b}$ Yerevan State University, Yerevan, Armenia
}

We report on specific signatures of one-mode squeezing due to multipulse control over quantum dynamics. As an important example, we discuss generation of squeezed bright light beams in optical parametric oscillator subjected to a periodic sequence of laser pulses. We show that the synchronized pulse control essentially improves the degree of integral squeezing, making it below the standard limit established for a stationary cw regime.

Исследованы свойства одномодовых сжатых состояний, которые обусловлены контролированием квантовой динамики посредством световых импульсов. В качестве примера рассмотрена генерация сжатых интенсивных световых пучков в оптическом параметрическом осцилляторе под действием периодической последовательности лазерных импульсов. Показано, что синхронизированный импульсный контроль существенно улучшает степень интегрального сжатия, делая его ниже стандартного предела, установленного для стационарного режима.

\section{INTRODUCTION}

Controlling quantum dynamics is one of the most fundamental issues in quantum physics, as well as in modern information processing. This problem is also connected with elaboration of quantum devices. In one of the standard techniques [1,2], control of optical quantum system is achieved through the application of suitable tailored, time-dependent external fields, including application of synchronized laser pulses. In this report, we want to extend pulses control strategy by considering generation of squeezed light in dissipative systems.

Squeezed states of light play an important role in developments of quantum physics. These states have been widely employed to achieve measurement sensitivity beyond the standard quantum limits in applications such as precision interferometry and atomic spectroscopy. Novel discoveries made with them include quantum information processing with continuous variables (CV) [3]. In particular, squeezed states of light have been used as a resource for quantum cryptographic protocols. Single-mode squeezed states have been also served as $\mathrm{CV}$ entanglement source since combining two single-mode squeezed states at a beam splitter creates an entangled two-mode state.

\footnotetext{
${ }^{1}$ E-mail: ngevorg@www.physdep.r.am

${ }^{2}$ E-mail: gkryuchk@www.physdep.r.am
} 
Optical parametric oscillators (OPO) based on processes of down conversion in a cavity have proved to be efficient sources of squeezed light [4]. Excellent agreement between theory and experiment of squeezing is obtained in the region below threshold for OPO and in the spectral domain. A systematic quantum theory of OPO including the near-threshold region has also been developed in (see [5] and references therein).

As is well known, the total or integral one-mode squeezing is usually analyzed through the variances $V(\theta)$ of quadrature amplitudes of electromagnetic field $X(\theta)=\frac{1}{\sqrt{2}}\left(a^{+} \mathrm{e}^{-i \theta}+a \mathrm{e}^{i \theta}\right)$, where $a, a^{+}$are the boson operators, $V(x)=\left\langle x^{2}\right\rangle-\langle x\rangle^{2}$ is a denotation of a variance. It should be noted that an integral squeezing for the intracavity mode of subharmonic generated by OPO reaches only $50 \%$ relative to the level of vacuum fluctuations, $1>V \geqslant 0.5 V_{0}$, and equality takes place if the pump field intensity is close to the generation threshold [5]. It is obvious that such a limitation on the degree of squeezing is due to dissipation and decoherence processes, as well as the cavity induced feedback.

In this paper we obtain a remarkable result that application of pump field with periodically varying amplitudes instead of $\mathrm{cw}$ pump field essentially improves the degree of squeezing in OPO, making it beyond the limit $50 \%$. We demonstrate this phenomenon for OPO in tworesonant optical ring cavity, concluding that such an essential improvement of total squeezing takes place for both below- and above-threshold operational regimes.

Noteworthy is the difference between the focus of our paper and most of the previous works devoted to the study of squeezing. It is an established standard to describe squeezing with the spectra of quantum fluctuations, as has been done even for some pulsed squeezing experiments [6]. Unlike that, we follow the ideology of time-resolved quantum information technologies which are now in the stage of development (see, for example, [7]), and analyze the integral squeezing characteristics of periodically pulsed light beams in the time domain.

\section{MEAN PHOTON NUMBER AND SQUEEZED VARIANCE}

We consider standard OPO in a cavity that supports the pump mode at frequency $\omega_{L}$ and subharmonic mode at $\omega_{L} / 2$. Down conversion of the pump photons to resonant subharmonicmode photons at frequency $\omega_{L} / 2$, i.e., the process $\omega_{L} \rightarrow \frac{\omega_{L}}{2}+\frac{\omega_{L}}{2}$, occurs due to a $\chi^{(2)}$ nonlinearity inserted in the cavity. The novelty is that pump mode is driven by an amplitudemodulated external field at the frequency $\omega_{L}$ with time periodic, real valued amplitude $f(t+$ $\tau)=f(t)$. Thus, equations describing this system are the well-known equations of an ordinary OPO which involves modulated amplitude $f(t)$ instead of time-independent amplitude for $\mathrm{cw}$ regime (see, for example, [5]).

The surprising thing is that in the semiclassical approach it is possible to find an analytical solution for the mean photon numbers and phases of generated modes provided that detunings of modes are equal to zero. Some details can be found in the paper [6] devoted to investigation of time-modulated nondegenerate OPO. In over-transient regime, if $t-t_{0} \gg \gamma^{-1}, \gamma_{3}^{-1}$, the mean photon number of subharmonic $\omega_{L} / 2$ reads as

$$
n_{c}^{-1}(t)=\frac{2 k^{2}}{\gamma_{L}} \int_{t_{0}}^{t} \exp \left(-2 \int_{\tau}^{t}\left(\frac{k}{\gamma_{L}} f\left(t^{\prime}\right)-\gamma\right) d t^{\prime}\right) d \tau
$$


while the phase of the subharmonic is equal to $\varphi=\phi_{L} / 2 \pm \pi m,(m=0,1,2, \ldots)$ and describes twofold symmetry in phase space. Here $\phi_{L}$ is a pump field phase, $\gamma$ and $\gamma_{3}$ are the decay rates of modes $\omega_{L} / 2$ and $\omega_{L}$. The constant $k$ is proportional to the $\chi^{(2)}$ susceptibility and determines an efficiency of the down-conversion process. Note that in this regime the photon number is a periodic function of time.

To calculate the squeezed variance $V$, we use an approach recently developed in analyzing of time-modulated NOPO [8]. This approach is based on perturbative analysis of stochastic equations of motion of time-modulated OPO on the small parameter of the theory $\lambda / \gamma=$ $k^{2} / \gamma \gamma_{3} \ll 1$. In the below-threshold regime, $\overline{f(t)}<f_{\text {th }}=\gamma \gamma_{L} / k$, the result is calculated in the following form:

$$
V(t)=\gamma \int_{-\infty}^{0} \exp \left(-2 \int_{\tau}^{0}\left(\gamma+\frac{k}{\gamma_{3}} f\left(t^{\prime}+t\right)\right) d t^{\prime}\right) d \tau
$$

while for time-modulated OPO above threshold, $\overline{f(t)}>f_{\mathrm{th}}$,

$$
V(t)=\gamma \int_{-\infty}^{0} \exp \left(-2 \int_{\tau}^{0}\left(\gamma+\frac{k}{\gamma_{3}} f\left(t^{\prime}+t\right)+\gamma n_{c}\left(t^{\prime}+t\right)\right) d t^{\prime}\right)\left[1+2 n_{c}(\tau+t)\right] d \tau .
$$

Here we have defined the period-averaged pump field amplitude $\bar{f}=\frac{1}{T} \int_{0}^{T} f(t) d t$. Particularly, for the case of monochromatic wave pump field, $f(t)=\bar{f}=$ const, Eq. (2) takes the form $V=\frac{\gamma \gamma_{3}}{2\left(\gamma \gamma_{3}+k \bar{f}\right)}$, which coincides with the well-known result for the ordinary OPO below threshold. Note that the results (2), (3) are different from analogous results obtained for two-mode squeezed variances [6].

\section{PULSED CONTROL OF SQUEEZING}

Let us now analyze the synchronized pulse application in the context of squeezed states. We consider OPO subjected to a periodic sequence of identical laser pulses. We consider a rectangular form of the pulses of the duration $T_{1}$, assuming that $T_{1}$ is much less than the interval $T_{2}$ between the pulses. In this case the period-averaged pump field amplitude $\bar{f}=f_{L} T_{1} /\left(T_{1}+T_{2}\right)$, where $f_{L}$ is the highness of laser pulses, and hence the above-threshold regime is realized if $f_{L} T_{1}>\frac{\gamma \gamma_{3}}{k}\left(T_{1}+T_{2}\right)$. The mean photon numbers and the squeezed variance $V(t)$ are calculated on formulas (1) and (2), (3). The predictions of the numerical calculations are shown in Figs. 1 and 2 for two preferable above-threshold regimes (for typical $\gamma=10^{6} \mathrm{~s}^{-1}, T_{1}=10^{-8} \mathrm{~s}$ and the repetition rate $T_{2}^{-1}=1 \mathrm{MHz}$ ). It is clearly evident from these figures that the mean photon number increases during laser pulses and decays during the interval $T_{2}$ between pulses due to dissipation in the cavity. One can conclude from Fig. 1, $b$ and Fig. 2, $b$ that the squeezing criterion $V<1$ is fulfilled for any time intervals. However, we have also found remarkable result that the variance goes below the level of $0.5 \%$ estabilished 
for an ordinary OPO in a steady state, i.e., $V \leqslant \frac{1}{2} V_{0}$, where the variance $V_{0}$ corresponds to the level of vacuum fluctuations and is normalized as $V_{0}=1 / 2$. Such a situation occurs for definite time intervals corresponding to the ranges of maximal photon numbers, and for the appropriate chosen parameters. Thus, high-degree squeezing takes place for nonstationary regime, if $T_{1}$ is enough shorter than the relaxation time and hence the dissipative effects in modes dynamics are still inessential. We also illustrate these results analytically by calculation of the minimum values $V_{\min }=V\left(t_{m}\right)$ at fixed time intervals $t_{m}=t_{0}+m T_{2}$. Considering for simplicity OPO below and near the threshold and assuming $T_{1} \ll T_{2}$, we get from Eq. (2)

$$
V_{\min }=\frac{1}{2} \mathrm{e}^{-2 \varepsilon_{L} T_{1}} \frac{1-\mathrm{e}^{-2 \gamma T_{2}}}{1-\mathrm{e}^{-2 \gamma T_{2}-2 \varepsilon_{L} T_{1}}},
$$

where $\varepsilon_{L}=f_{L} k / \gamma_{3}$. This formula is in accordance with the data of Fig. 1, $b$ and Fig. 2, $b$. The degree of integral squeezing is seen to increase with the parameter $\varepsilon_{L} T_{1}$.
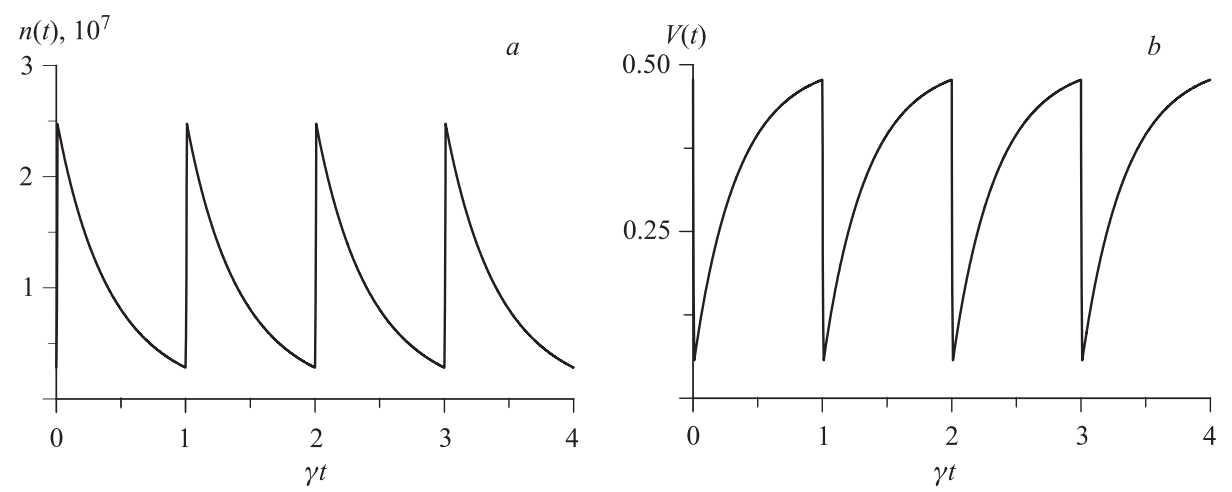

Fig. 1. Mean photon number $(a)$ and the variance $(b)$ versus dimensionless time for the parameters: $k^{2} / \gamma_{L} \gamma=10^{-8}, T_{1}=0.01 \gamma^{-1}, T_{2} / T_{1}=100, f_{L}=1.1 \frac{\gamma \gamma_{3}}{k}\left(1+T_{2} / T_{1}\right)$
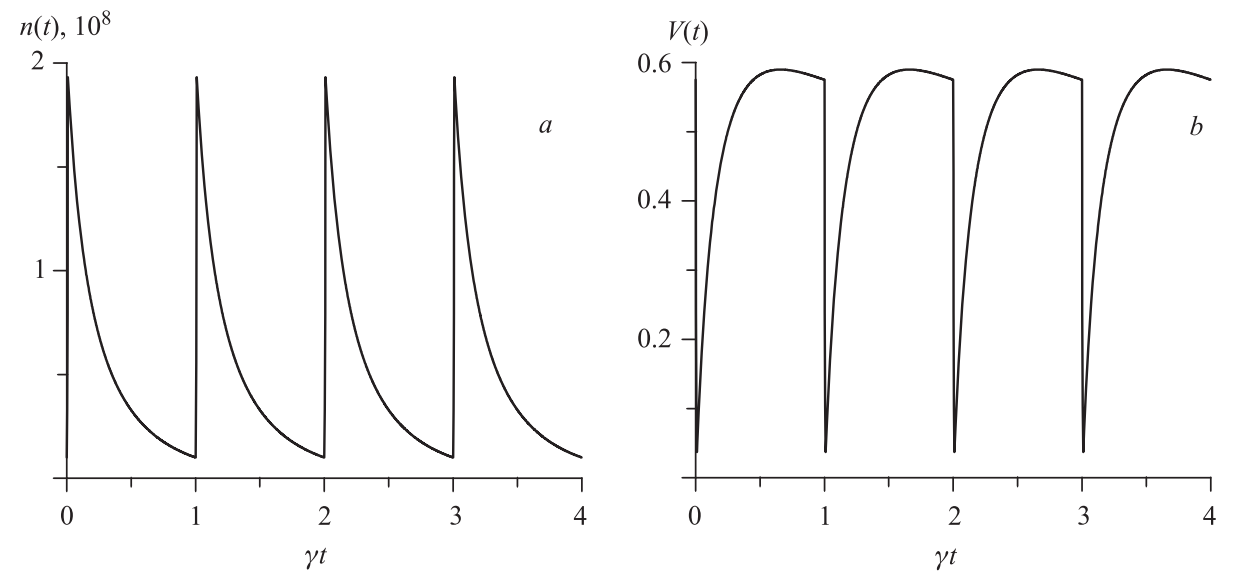

Fig. 2. Mean photon number $(a)$ and the variance $(b)$ versus dimensionless time for the parameters: $k^{2} / \gamma_{L} \gamma=10^{-8}, T_{1}=0.01 \gamma^{-1}, T_{2} / T_{1}=100, f_{L}=1.5 \frac{\gamma \gamma_{3}}{k}\left(1+T_{2} / T_{1}\right)$ 
288 Adamyan H. H., Gevorgyan N. T., Kryuchkyan G. Yu.

\section{CONCLUSION}

We have demonstrated the synchronized pulse control over squeezing in OPO. On the whole the integral variance of squeezed quadrature goes below the level $50 \%$ relative to the level of vacuum fluctuations, $V<1 / 4$, in contrast to a usual OPO operated with cw pump. We expect that an experimental evidence of pulsed squeezed light may be given the time domain as it has been demonstrated for time resolved homodyne squeezing measurement [7].

Acknowledgements. This work was supported by ANSEF, grant No.05-PS-compsci-8966, and INTAS, grant No. 04-77-7289.

\section{REFERENCES}

1. Viola L., Lloyd S. // Phys. Rev. A. 1998. V.58. P. 2733.

2. Vitali D., Tombesi P. // Phys. Rev. A. 1999. V.59. P. 4178.

3. Braunstein S.L., Pati A.K. Quantum Information Theory with Continuous Variables. Dordrecht: Kluwer, 2003.

4. Wu L.A. et al. // Phys. Rev. Lett. 1986. V.57. P. 2520.

5. Chaturvedi S., Dechoum K., Drummond P. D. // Phys. Rev. A. 2002. V.65. P. 033805.

6. Slusher R. E. et al. // Phys. Rev. Lett. 1987. V.59. P. 2566.

7. Wenger J., Tualle-Brouri R., Grangier Ph. // Opt. Lett. 2004. V.29. P. 1267.

8. Kryuchkyan G., Adamyan H. Strong Entanglement of Bright Light Beams in Controlled Quantum Systems // NATO Series. V. Decoherence, Entanglement and Information Protection in Complex Quantum Systems. Springer; Kluwer, 2005. P. 105-126. 